\title{
AlGaN Heterostructure Optimization for Photodetectors
}

\author{
S.I. Didenko, O.I. Rabinovich, S.A. Legotin, I.V. Fedorchenko, A.A. Krasnov, Yu.V. Osipov, M.S. Melnik, \\ K.A. Sergeev
}

\author{
NUST "MISiS”, 4, Leninskiy prosp., 119040 Moscow, Russia
}

(Received 04 May 2016; published online 03 October 2016)

\begin{abstract}
GaN-based photodetectors are of great interest for applications involving detection and imaging in the UV-visible wavelength range. Most of experimental and theoretical work in this area has been focused so far on Schottky diode and $p-i-n$ structures for solar blind applications. However, it seems that bipolar phototransistors with their additional functionality could also be of potential interest. In this work we present the results of phototransistors parameters simulation using the software - Sim Windows. The structure analyzed consisted, counting from the substrate, of $n$ - $\mathrm{Al}_{x} \mathrm{Ga}_{1-x} \mathrm{~N}$ collector, $p$-GaN base and $n-\mathrm{Al}_{x} \mathrm{Ga}_{1-x} \mathrm{~N}$ emitter. The $\mathrm{Al}$ mole fraction in the collector and emitter was varied from $x=0.2$ to $x=0.3$. The collector and emitter thickness was taken as $0.9 \mu \mathrm{m}$. The doping level in the emitter and collector was varied from $10^{17}$ to $10^{19} \mathrm{~cm}^{-3}$. The $p$-GaN base thickness was taken as $0.3 \mu \mathrm{m}$, with doping of $10^{17}-10^{18} \mathrm{~cm}^{-3}$. The electron and hole lifetimes in the base were taken as $12 \mathrm{~ns}$.
\end{abstract}

Keywords: Photodetector, Simulation, Nanoheterostructure, Nitride.

DOI: $10.21272 /$ jnep.8(3).03036

PACS numbers: 00.05.Tp, 85.60.Jb

\section{INTRODUCTION}

In this paper an actual problem of creating semiconductor photodetectors for the ultraviolet spectral range is discussed. Such devices can be widely used in various fields of technology, including devices that analyze the gases and liquids composition, and in the open optical communication lines with increased noise immunity in high solar radiation.

One of the most promising ways to create UV photodiodes is $\mathrm{Al}_{x} \mathrm{Ga}_{1-x} \mathrm{~N}$ heterostructures usage and the base technology of their growth is metal organic chemical vapour deposition compounds (MOCVD), which allows producing heterostructures with high performance. Theoretically, these heterostructures allows creating photodiodes in the wavelength range 220-350 $\mathrm{nm}$. Now it has been developed and manufactured a photodiode AG38S-SMD with sensitivity $0.12-0.16 \mathrm{~A} / \mathrm{cm}^{2}$ for the radiation wavelength range of 250-350 $\mathrm{nm}$ [1].

Our investigations based on computer simulation showed that, based on such heterostructures not only photodiodes can be produced but also phototransistors (PT) with a sensitivity of more than $100 \mathrm{~A} / \mathrm{cm}^{2}$, in this wavelengths area [2-7]. It has been proposed ultraviolet phototransistor (UV PT) structure, based on the capabilities of this technology with GaN and AlGaN collector. In this paper it is investigated the first step of the PT development - defining the multilayer heterostructures design by computer simulation: the structure type $(n-p-n$ or $p-n-p)$, the aluminum content, dopants thickness and concentration in the layers structure and other parameters.

\section{EXPERIMENTAL PROCEDURES}

$\mathrm{Al}_{x} \mathrm{Ga}_{1-x} \mathrm{~N}$ phototransistor simulation with disabled base. SimWindows software (SW) was used in current investigation [6]. Main features of the software and method of its usage for semiconductor devices simulation have been described in [2-7].

For $\mathrm{Al}_{x} \mathrm{Ga}_{1-x} \mathrm{~N}$ photodiodes and phototransistors simulation special materials and devices files were created. Each individual Het. in its device file contained characteristics, such as the geometric dimensions of the emitters, quantum wells and barriers, the quantum well and barrier numbers, the solid solution content, the conductivity type, concentration, and the activation energy of dopants in each of the Het. areas. In the materials file for solid solutions, more than 25 parameters such as the band gap, refractive index, optical absorption, thermal conductivity, mobility and lifetime of charge carriers, electron affinity, the coefficients of radiative and non-radiative recombination, etc. were included.

Figure 1 shows the energy band diagram of a symmetric structure $n$-AlGaN/p-GaN/n-AlGaN.

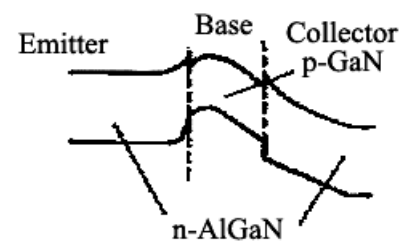

Fig. 1 - The current-voltage characteristic of a phototransistor double heterojunction

This device is characterized by high values of gain for both shift polarities. Effective injection capacity is provided by the fact that the $p$-GaN base layer has a narrower bandgap than the emitter and collector layers. Thus delete limitation of conventional transistors eliminated. The limitation consistence of the need to create a light doped base layer and it can be realized the conditions under which the depletion region of reverse-shifted transition is mainly concentrated in the collector layer. The device has a high gain.

During heterostructure simulation for high efficiency and high sensitive phototransistor the structure consisted of the $p$ - $\mathrm{Al}_{0.3} \mathrm{Ga}_{0.7} \mathrm{~N}$ collector, $n-\mathrm{Al}_{0.3} \mathrm{Ga}_{0.7} \mathrm{~N}$ emitter with an aluminum content of $30 \%$ and a pGaN base. The emitter and collector thickness was 
$0.875 \mu \mathrm{m}$, the base thickness $-0.3 \mu \mathrm{m}$. Acceptor concentration in $p-\mathrm{Al}_{0.3} \mathrm{Ga}_{0.7} \mathrm{~N}$ collector was $10^{17} \mathrm{~cm}^{-3}$, donors in $n$-Alo.3Ga0.7 $\mathrm{N}$ emitter was $10^{17} \mathrm{~cm}^{-3}$, the acceptor concentration in the $p$-GaN base $-10^{17} \mathrm{~cm}^{-3}$ or $10^{18} \mathrm{~cm}^{-3}$. The lifetimes values of the nonequilibrium electrons and holes in all areas of PTs were equal to $50 \mathrm{~ns}$. The device files have been created for both the concentration of acceptors in the base.

Method for PT characteristics simulation at two acceptor concentrations in PTs base $\left(N_{a}=10^{17} \mathrm{~cm}^{-3}\right.$ or $\left.N_{a}=10^{18} \mathrm{~cm}^{-3}\right)$ was:

For the dark current density value $J_{\mathrm{Ph}}, \mathrm{A} / \mathrm{cm}^{2}$ determination at different voltage $\mathrm{U}$ between the PT emitter and collector (plus on $p-\mathrm{Al}_{0.3} \mathrm{Ga}_{0.7} \mathrm{~N}$ collector), the voltage range was $U=(1-9) \mathrm{V}$.

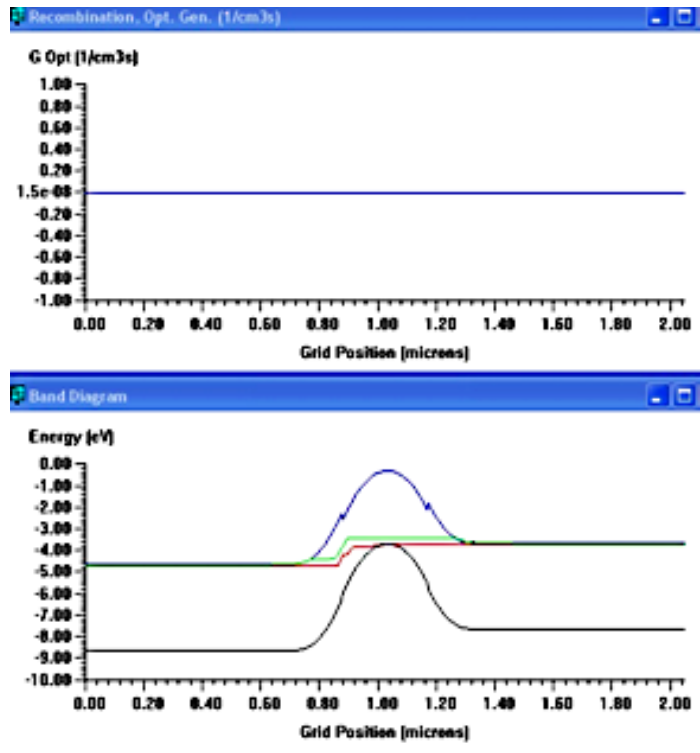

Fig. 2 - Phototransistor simulation without illumination at the acceptor concentration in the base $-N_{a}=10^{17} \mathrm{~cm}^{-3}, U=1 \mathrm{~V}$

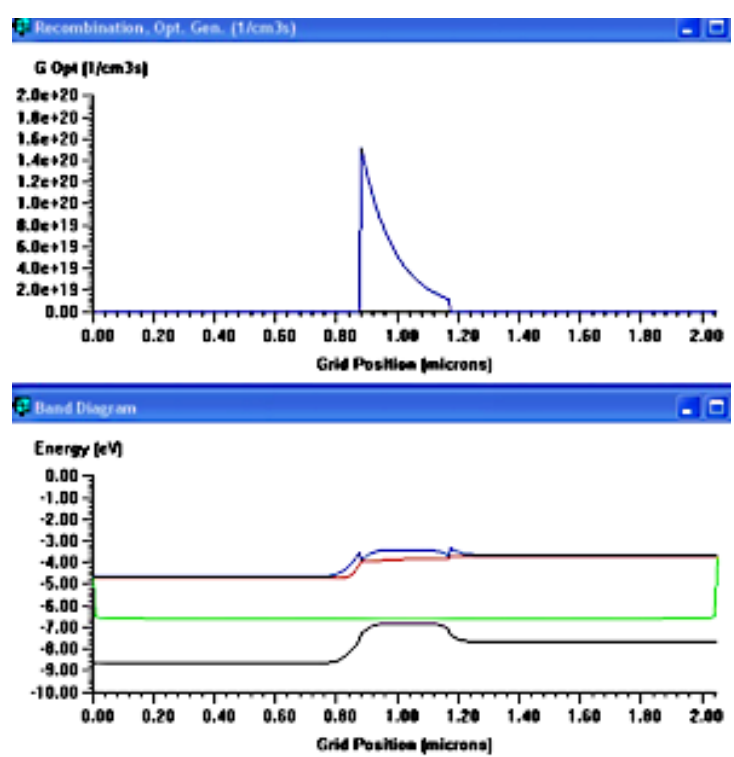

Fig. 3 - Phototransistor simulation at acceptor concentration in base $-N_{a}=10^{17} \mathrm{~cm}^{-3}$ and lighting power $P=1 \mathrm{~mW} / \mathrm{cm}^{2}, U=1 \mathrm{~V}$
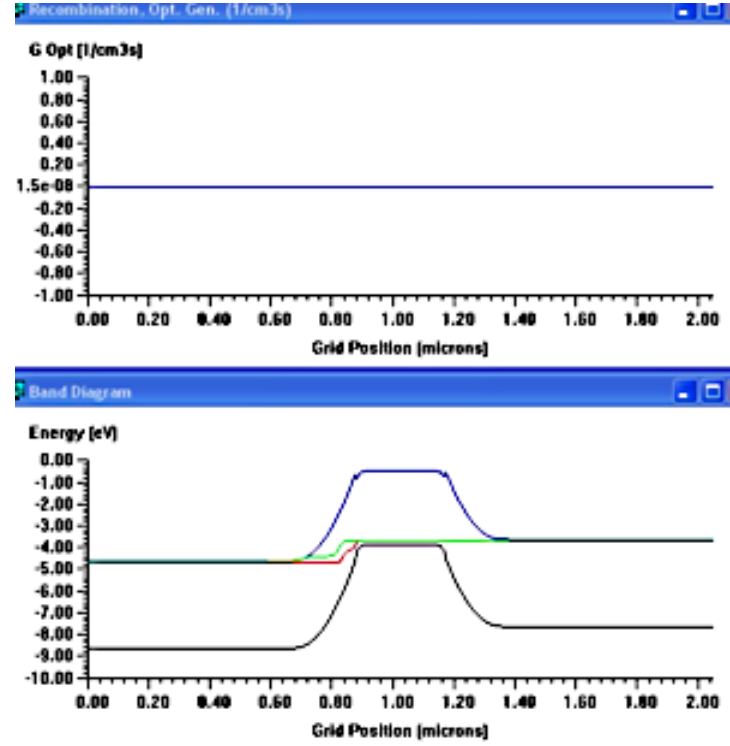

Fig. 4 - Phototransistor simulation without illumination at the acceptor concentration in the base $-N_{a}=10^{18} \mathrm{~cm}^{-3}, U=1 \mathrm{~V}$

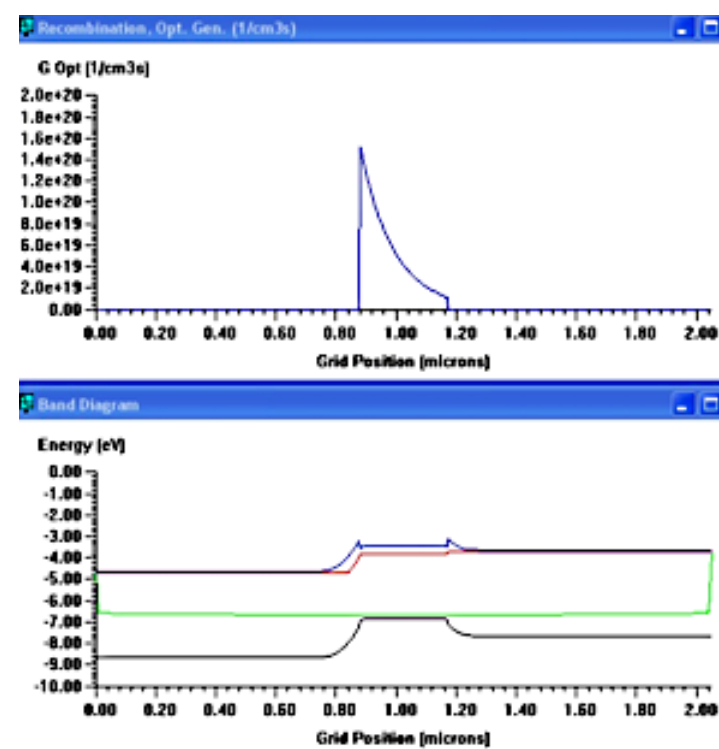

Fig. 5 - Phototransistor simulation at acceptor concentration in base $-N_{a}=10^{18} \mathrm{~cm}^{-3}$ and lighting power $P=1 \mathrm{~mW} / \mathrm{cm}^{2}$, $U=1 \mathrm{~V}$

For the photocurrent density value Jf, $\mathrm{A} / \mathrm{cm}^{2}$ determination at various lighting power density values $P$, W/cm ${ }^{2}$ was $P=\left(10^{-6}-10^{-1}\right) \mathrm{W} / \mathrm{cm}^{2}$ and voltage between the PT emitter and the collector (plus on $p$-Al $\mathrm{Al}_{0.3} \mathrm{Ga}_{0.7} \mathrm{~N}$ collector) was $U=(1-9) \mathrm{V}$. The energy of quanta during lighting was $E=3.5 \mathrm{eV}$. The $\mathrm{Al}$ mole fraction in the collector and emitter was varied from $x=0.2$ to $x=0.3$. Figures 2-5 show the main characteristics of the phototransistor: Energy diagram of the structure and Recombination rate. The radiation is added on the left the structure surface ( $p$ - $\mathrm{Al}_{0.3} \mathrm{Ga} 0.7 \mathrm{~N}$ collector).

\section{RESULTS AND DISCUSSION}

Dark current density $J_{\mathrm{Ph}}, \mathrm{A} / \mathrm{cm}^{2}$ dependence at the voltage on PT is shown in Fig. 6. 


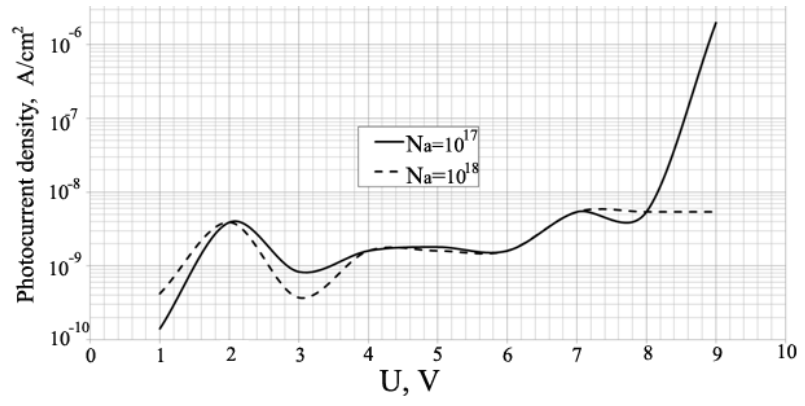

Fig. 6 - Dark current density $j_{\text {ph }}$ vs the PT voltage

It is clearly seen that in the PTs at a acceptors concentration in the base $N_{a}=10^{17} \mathrm{~cm}^{-3}$, dark current begins to rise sharply at a voltage greater than 8 volts, which is obviously corresponds with start of the clamping of the emitter and collector (Early's effect). When the acceptor concentration in the base $N_{a}=10^{18} \mathrm{~cm}^{-3}$, this effect occurs at higher voltages. At voltages less than 8 volts dark current density does not exceed $10^{-8} \mathrm{~A} / \mathrm{cm}^{2}$. This value limits the minimum magnitude of photocurrent density and, consequently, the minimum value of the recorded radiation power.

Photocurrent density $j_{\mathrm{F}}, \mathrm{A} / \mathrm{cm}^{2}$ dependence at the light power density $P, \mathrm{~W} / \mathrm{cm}^{2}$ at a constant voltage $U$ between the PTs emitter and the collector are shown in Fig. 7-9. Lighting power range was $P=\left(10^{-6}-10^{-1}\right)$ $\mathrm{W} / \mathrm{cm}^{2}$; the voltage range was $U=(1-9) \mathrm{V}$. Due to huge data, it is presented by three sub-range voltage (1-3) (4-6) and the (7-9) V.

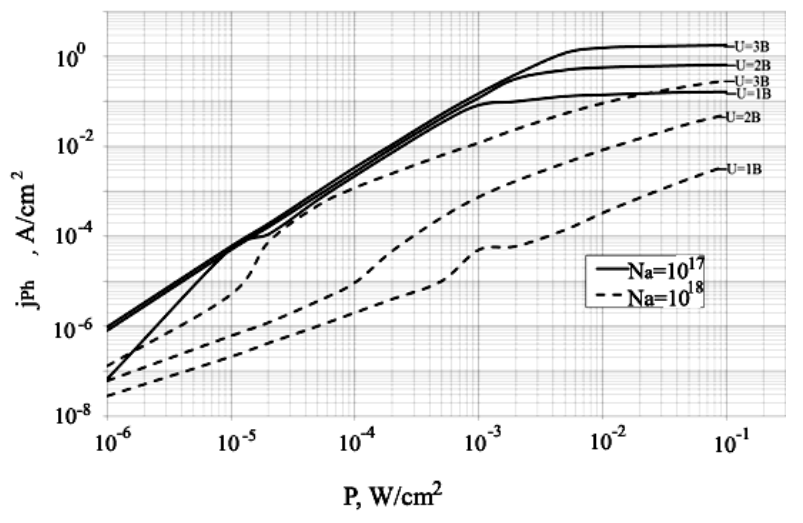

Fig. 7 - Photocurrent density $j_{\mathrm{F}}$ vs irradiation power $P, U=1-3 \mathrm{~V}$

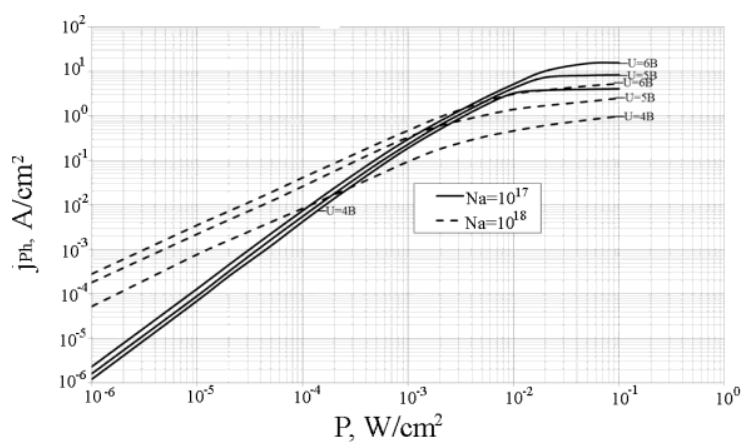

Fig. 8 - Photocurrent density $j_{\mathrm{F}}$ vs irradiation power $P, U=4-6 \mathrm{~V}$

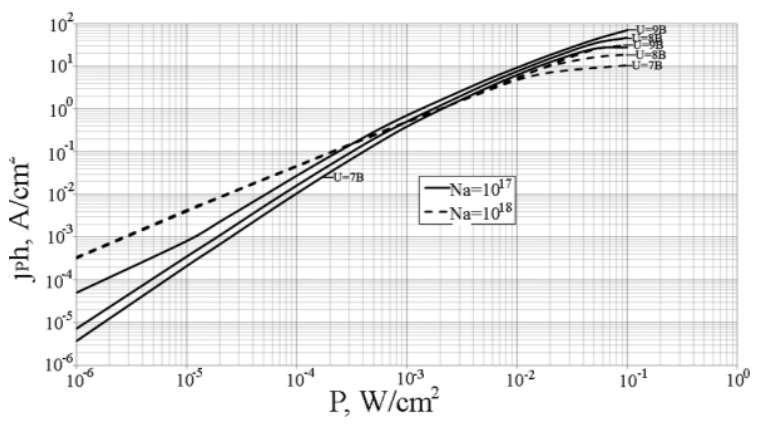

Fig. 9 - Photocurrent density $j_{\mathrm{F}}$ vs irradiation power $P, U=7-9 \mathrm{~V}$

Figure 10 clearly shows that at the acceptors concentration in the base $N_{a}=10^{18} \mathrm{~cm}^{-3}$, the PTs sensitivity has a large value in a wide range of lighting, especially in the region of small power values $P$.

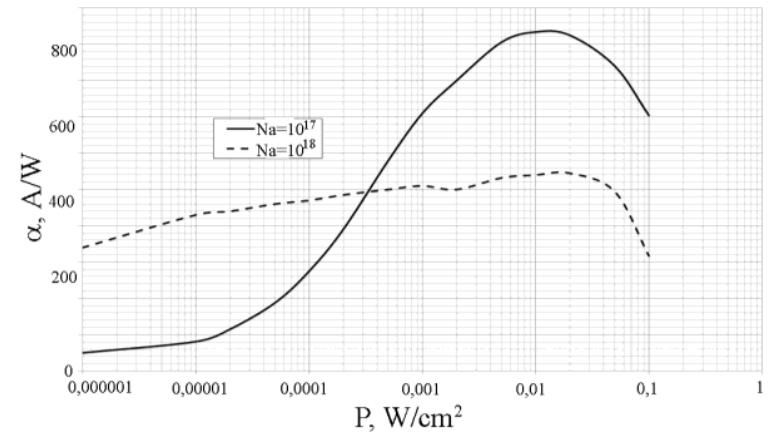

Fig. 10 - Phototransistor sensitivity vs light power $U=9 \mathrm{~V}$

At the same time, at the acceptor concentration in the base $N_{a}=10^{17} \mathrm{~cm}^{-3}$ the PT sensitivity is maximum at relatively larger values of the power $P$, which makes them very promising in a variety of applications.

Figure 11 shows the of the PT sensitivity dependence at voltage in the range from 1 to 9 volts at a power density of light $1 \mathrm{~mW} / \mathrm{cm}^{2}$, which is typical in many applications of UV photodetectors.

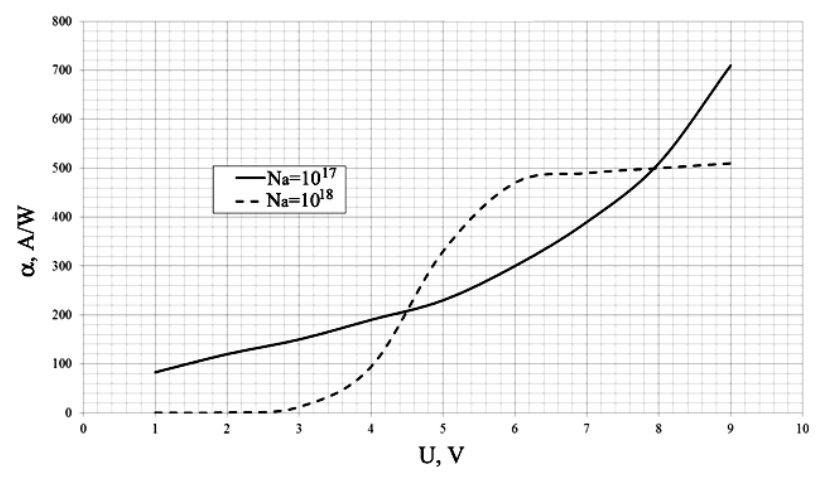

Fig. 11 - Phototransistor sensitivity vs voltage, the power illumination density of $1 \mathrm{~mW} / \mathrm{cm}^{2}$

Conclusion of this dependence is quite obvious - to obtain a high sensitivity at voltage, applied to the PT, must be in the range from 6 to 9 volts. At the end of the discussion of UV phototransistor characteristics simulating we present data of the PTs sensitivity spectral dependence (Fig. 12). 


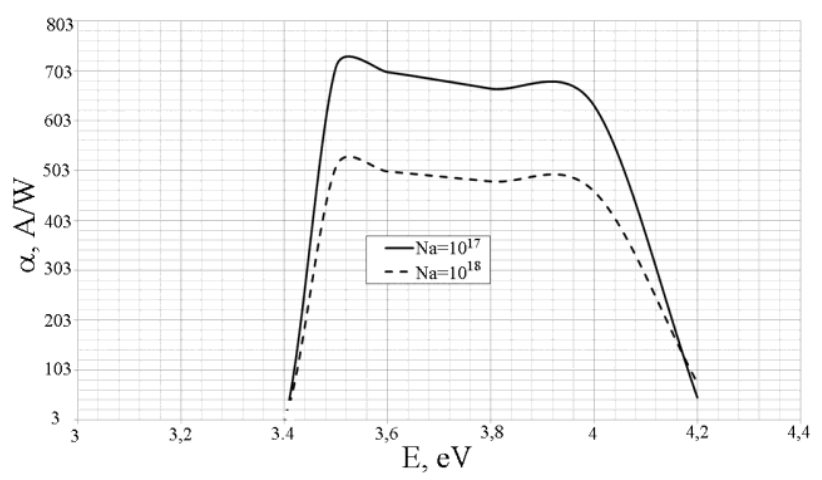

Fig. 12 - Phototransistor sensitivity vs quanta energy $E, \mathrm{eV}$ $P=10^{-3} \mathrm{~W} / \mathrm{cm}^{2}$ and $U=9 \mathrm{~V}$

It is clearly seen that the PTs sensitivity is very high in the range of photon energies from $3.5 \mathrm{eV}$ to $4 \mathrm{eV}$ (wavelength range from $354 \mathrm{~nm}$ to $309 \mathrm{~nm}$ ), which allows to use them as selective photodetectors. PTs selectivity can be increased by reducing the aluminum concentration in the $p$ - $\mathrm{Al}_{0.3} \mathrm{Ga}_{0.7} \mathrm{~N}$ collector up to $20 \%$. Sensitivity also can be increased by improving MOCVD technology for more high quality $\mathrm{AlGaN}$ multilayer structures (with minimum defect concentration). If the

\section{REFERENCES}

1. Sg Lux GmbH company. http://sglux.de/en/productcategory/sic-uv-photodiodes/

2. O.I. Rabinovich, J. Alloy. Compd. 586, S258 (2014).

3. O.I. Rabinovich, V.P. Sushkov, Semiconductors 43 No 4, 524 (2009).

4. O. Rabinovich, S. Legotin, S. Didenko, E. Yakimov, Yu. Osipov, I. Fedorchenko, Jpn. J. Appl. Phys. 55, 05FJ131 sapphire substrate could be replaced by a gallium nitride substrate grown on sapphire, the lifetimes of nonradiative recombination in the $\mathrm{PTs}$ base will be increased significantly.

\section{SUMMARY}

1. $\mathrm{Al}_{x} \mathrm{Ga}_{1-x} \mathrm{~N}$ heterostructure $\mathrm{PT}$ have a wide linear dynamic range of characteristics and the ability to control ultimate energy quantum cutoff. Due to this, $\mathrm{Al}_{x} \mathrm{Ga}_{1-x} \mathrm{~N}$ heterostructures are the most promising material for semiconductor UV - detectors.

2. The $\mathrm{Al}_{x} \mathrm{Ga}_{1-x} \mathrm{~N}$ heterostructures characteristics computer simulation for the most important types of UV photodetectors: photodiode with p-n junction and the phototransistor was carried out. The possibility of obtaining their high sensitivity and selectivity in a wide wavelength range was shown.

3. A new phototransistor structure with disabled base on the basis of a symmetrical heterostructure $n$ $\mathrm{Al}_{0.3} \mathrm{Ga}_{0.7} \mathrm{~N}-p \mathrm{GaN}-n \mathrm{Al}_{0.3} \mathrm{Ga}_{0.7} \mathrm{~N}$ was suggested. Simulation shows the possibility of creating a device with a sensitivity of 500-700 A/W based on structures with electrical and structural parameters, typical for serial production.

(2016).

5. D.W. Winston, SimWindows 1.5. Users manual (Colorado: 1999).

6. Wei Yang, High Gain GaN/AlGaN heterojunction Phototransistor, patent US006137123A, Oct. 24 (2000).

7. V.P. Sushkov, E.N. Vigdorovich, Abstracts IV RussianJapanese workshop. 659 (2006). 